\title{
Análise da aderência do disclosure da gestão de riscos pelas instituições financeiras à circular Bacen 3.477
}

On the acquisition of disclosure on risk management by financial institutions to the Bacen 3.477 notice

Análisis de la adherencia del disclosure de la gestión de riesgos por las instituciones financieras a la circular 3.477 de Bacen

\section{Mônica Campos Torres}

Mestre em Administração de Empresas pela Fucape

Diretora de Riscos e Controle - Banestes

Endereço: Av. Fernando Ferrari, n 1358, Bairro Boa Vista

CEP: 29075-505 - Vitória/ES - Brasil

E-mail: monicatorres@banestes.com.br

Telefone: (27) 4009-4444

\section{Fernando Caio Galdi}

Doutor em Ciências Contábeis pela Universidade de São Paulo

Professor Associado da Fucape

Endereço: Av. Fernando Ferrari, n 1358, Bairro Boa Vista

CEP: 29075-505 - Vitória/ES - Brasil

E-mail: fernando.galdi@fucape.br

Telefone: (27) 4009-4444

Artigo recebido em 23/04/2013. Revisado por pares em 13/09/2013. Reformulado em 26/10/2013. Recomendado para publicação em 29/11/2013 por Sandra Rolim Ensslin (Editora Científica). Publicado em 15/12/2013. 


\title{
Resumo
}

O Banco Central do Brasil publicou a Circular n. 3.477/09 que dispõe sobre a obrigatoriedade da divulgação de informações referentes à gestão de riscos, ao Patrimônio de Referência Exigido e ao Patrimônio de Referência. Este estudo verifica o nível de divulgação dessas informações pelas instituições financeiras, à luz das diretrizes da Circular ora citada. Os resultados demonstram que somente $49,5 \%$ das instituições divulgaram as informações no formato requerido pela norma. $\mathrm{O}$ tamanho, o nível de exposição a derivativos e o índice de alavancagem foram as variáveis que apresentaram relação estatística significativa, com uma relação negativa para esta última variável.

Palavras-chave: Disclosure. Instituições Financeiras. Gestão de Risco.

\begin{abstract}
The Central Bank of Brazil published the notice number 3.477/09 that reports on the requirement to make public information related to risk management, to both Patrimonio de Referência Exigido and to Patrimônio de Referência. This study observes the level of publicizing on this type of information by the financial institutions, taking into account the guidelines presented in the notice mentioned above. Results demonstrate that only $49.5 \%$ of institutions published information as requested by the norm. The size, the level of exposure to derivatives, and the level of financial leverage were the variables that presented significant statistical results, in a negative relation to the level of financial leverage.
\end{abstract}

Keywords: Disclosure. Financial institutions. Risk management.

\section{Resumen}

Banco Central de Brasil emitió la Circular no 3.477/09 que establece la obligación de divulgar la información relativa a la gestión de riesgos, al Capital de Solvencia Obligatorio y al Patrimonio de Referencia. Este estudio evalúa el nivel de divulgación de estas informaciones por parte de las instituciones financieras a la luz de las directrices de la circular mencionada. Los resultados muestran que sólo el $49,5 \%$ de las instituciones dio a conocer las informaciones requeridas por la circular. El tamaño, el nivel de exposición a los derivados financieros y el índice de apalancamiento fueron las variables que presentaron una relación estadísticamente significativa, con una relación negativa de esta última variable.

Palabras clave: Disclosure. Instituciones Financieras. Gestión de Riesgo. 


\section{Introdução}

Barth et al. (2001) comentam que o amplo escopo das crises bancárias, bem como evidências objetivas do sistema bancário no desenvolvimento econômico, geraram demandas para reformas na regulação e supervisão das instituições financeiras. Dentro desse contexto se inserem instituições como o Comitê da Basileia sobre a Supervisão Bancária (Basel Committee on Banking Supervision - BCBS), o Fundo Monetário Internacional (FMI) e o Banco Mundial (BM), que promovem extensas listas de melhores práticas a serem adotadas pelos países e seus bancos de maneira a fortalecer o sistema bancário. Neste sentido, diversos países uniram esforços no sentido de estabelecer diretrizes a serem observadas pelas Instituições Financeiras (IF), que visam mitigar riscos e evitar possíveis perdas. O Comitê da Basileia sobre a Supervisão Bancária (Basel Committee on Banking Supervision - BCBS) surgiu em meio à falência e internacionalização crescente de bancos com o objetivo de melhorar a qualidade da supervisão bancária em todo o mundo instituindo um sistema padrão de mensuração dos requerimentos mínimos de capital (BCBS, 1988).

A regulamentação sobre o ambiente de controle e gestão de riscos em IF vem passando por profundas atualizações desde a publicação do Acordo de Capital de Basileia (1988) - International Convergence of Capital Measurement and Capital Standards, ou Basileia I e principalmente com a publicação no ano de 2004 do Novo Acordo de Capital de Basileia (Basileia II). O Basileia II, adotado por mais de 100 países, propõe um conjunto mínimo de diretrizes para adequação de capital das IF e introduz inovações, dentre elas dois novos pilares, o Processo de Revisão de Supervisão Bancária - Pilar II e Disciplina de Mercado - Pilar III. (BCBS, 2004).

O Pilar III, foco deste estudo, estabelece requerimentos de divulgação de informações relativas ao grau de exposição a risco pelas IF, visando ao exercício da disciplina de mercado, por meio da transparência, e a redução da assimetria da informação. A importância da transparência nos mercados financeiros é enfatizada por Goulart e Carvalho (2004, p. 13) ao afirmarem que "à medida que o sistema financeiro torna-se mais transparente, o mercado acaba exercendo uma "pressão", ou uma espécie de fiscalização, no sentido de que cada IF detenha "níveis de capital consoantes com seu perfil de risco"(GOULART;CARVALHO, 2004, p. 13).

Desde a aprovação do Basileia II, a divulgação de informações por parte das IF tem apresentado uma evolução ao longo dos anos, mas que ainda não é significativa diante das recomendações do Pilar III do Basileia II, conforme resultados de diversos estudos com enfoque na avaliação do nível de divulgação praticado pelas IF no Brasil (por exemplo, GOULART; CARVALHO, 2004; XAVIER, 2003; BARROSO; LUSTOSA; MORAES, 2004; ALVES, 2005; COSTA; GOLDNER; GALDI, 2007; DI BENEDITTO; SILVA, 2007; DOORNIK et al., 2007; ANDRADE; NIYAMA; SANTANA, 2009; DANTAS et al., 2010).

Dantas et al. (2005) afirmaram que independente do nível de desenvolvimento do mercado em que as empresas operam, existe a necessidade de reguladores estabelecerem as informações que devem ser divulgadas, pois relatam diversos estudos que mostram que as empresas apresentam muita resistência em aumentar o nível de divulgação financeira sem que haja uma pressão dos reguladores.

O Banco Central do Brasil (BACEN), principal regulador das IF, adotou as recomendações do Comitê de Basileia desde o Basileia I, por meio da Resolução 2.099 de 1994, ao estabelecer que as instituições autorizadas a operar no Brasil devem constituir um

139 ISSN 2175-8069, UFSC, Florianópolis, v. 10, n. 21, p. 137-158, set./dez. 2013 
valor de Patrimônio Líquido Exigido (PLE) maior ou igual a 8\% de seus ativos ponderados por fatores de risco. Índice este alterado para 11\% no Brasil em 1997.

Mais especificamente no que tange à divulgação de informações e em linha com as diretrizes do Pilar III, o BACEN publicou, em dezembro de 2009, a Circular n. 3.477 que dispõe sobre a obrigatoriedade de IF divulgarem informações referentes à gestão de riscos, ao Patrimônio de Referência Exigido (PRE) e à adequação do Patrimônio de Referência (PR).

Diante do contexto abordado, procurou-se, no decorrer deste estudo, responder à seguinte questão: As Instituições Financeiras que possuem maior exposição a riscos apresentam maior aderência à Circular BACEN 3.477?

Essa normativa representa a referência base deste estudo que tem dois objetivos principais: (i) identificar as variáveis determinantes de um maior nível de divulgação dos requisitos requeridos pela Circular BACEN 3.477 e (ii) verificar o nível de aderência das IF a esses requisitos.

Este trabalho se torna relevante do ponto de vista do regulador do sistema financeiro nacional na medida em que avalia a adequação dos bancos atuantes no Brasil à nova norma $\mathrm{e}$ apresenta oportunidades de melhoria e/ou adaptações para sua melhor aplicação. Do ponto de vista de relevância à comunidade acadêmica, o presente trabalho identifica fatores idiossincráticos das empresas que podem contribuir com uma maior ou menor aderência a um determinado normativo. Assim, os fatores identificados podem contribuir com pesquisas futuras que venham a avaliar a aderência aos requisitos de normativos no mercado bancário brasileiro.

Para o alcance desses objetivos, as informações foram coletadas em documentos de divulgação relativos à gestão de riscos, na data-base Dezembro/2010, em sítios eletrônicos, principalmente nas seções "Institucional", "Relação com Investidores", "Governança Corporativa" e "Gerenciamento de Riscos", das IF participantes do "Consolidado Bancário I", constantes do relatório BACEN "50 Maiores Bancos e o Consolidado do Sistema Financeiro Nacional". ${ }^{1}$

\section{Referencial Teórico}

A revisão teórica tem como objetivo resgatar os principais conceitos que sustentam o problema de pesquisa, as variáveis e as hipóteses propostas neste estudo.

\subsection{Risco}

Assumir riscos é inerente aos processos de uma instituição financeira. Estas devem se assegurar que estão adotando controles internos eficientes que os avaliem e os administrem adequadamente.

Risco para o Committee of Sponsoring Organizations of the Treadway Comission (COSO ERM, 2004, p. 52), é "a possibilidade de que um evento ocorra e prejudique a realização dos objetivos."

Já para o Instituto Brasileiro de Governança Corporativa (IBGC), em seu Guia de Orientação para Gerenciamento de Riscos Corporativos (2007, p. 11), risco significa "a

\footnotetext{
${ }^{1}$ Apesar da denominação do relatório reportar a apenas 50 Instituições Financeiras, o mesmo é composto de 138, agrupadas em Consolidado Bancário I, II e IV. 
possibilidade de algo não dar certo, mas seu conceito atual envolve a quantificação e qualificação da incerteza, tanto no que diz respeito às perdas como aos ganhos."

São várias as abordagens existentes sobre o conceito de risco, mas este estudo tratará dos riscos inerentes aos negócios financeiros, tais como risco de crédito, de mercado, operacional e liquidez, definidos pelo BACEN, respectivamente, por meio das resoluções 3.721 (2009), 3.464 (2007), 3.380 (2006) e 2804 (2000).

O risco de crédito é definido como a possibilidade de se ocorrer "perdas associadas ao não cumprimento pelo tomador ou contraparte de suas respectivas obrigações financeiras nos termos pactuados, à desvalorização de contrato de crédito decorrente da deterioração na classificação de risco do tomador, à redução de ganhos ou remunerações, às vantagens concedidas na renegociação e aos custos de recuperação."

Já o risco de mercado é definido pelo BACEN como a "possibilidade de ocorrência de perdas resultantes da flutuação nos valores de mercado de posições detidas por uma instituição financeira". Complementa esse conceito esclarecendo que ele "inclui os riscos das operações sujeitas à variação cambial, das taxas de juros, dos preços de ações e dos preços de mercadorias (commodities)."

O risco operacional é definido pelo BACEN (2006) como "a possibilidade de ocorrência de perdas resultantes de falha, deficiência ou inadequação de processos internos, pessoas e sistemas, ou de eventos externos." (BACEN, 2006).

O risco de liquidez é definido pelo BACEN (2000) como:

[...] ocorrência de desequilíbrios entre ativos negociáveis e passivos exigíveis 'descasamentos' entre pagamentos e recebimentos - que possam afetar a capacidade de pagamento da instituição, levando-se em consideração as diferentes moedas e prazos de liquidação de seus direitos e obrigações. (BACEN, 2000).

\subsection{Do "Basileia I" ao "Basileia II"}

O Comitê da Basileia de Supervisão Bancária (BCBS) foi criado em 1975 pelos presidentes dos bancos centrais dos países do Grupo dos Dez (G-10). Ele é composto de autoridades de supervisão bancária e representantes dos bancos centrais da Bélgica, Canadá, França, Alemanha, Itália, Japão, Luxemburgo, Países Baixos, Espanha, Suécia, Suíça, Reino Unido e Estados Unidos, com sede no Bank for International Settlements (BIS), na cidade de Basileia, Suiça. (BCBS, 1988).

O BIS é uma instituição internacional que tem como objetivo promover a cooperação entre os Bancos Centrais, atuando como um Banco Central dos Bancos Centrais e, por meio do Comitê de Basileia Supervisão Bancária da Basileia, analisa e discute sobre as melhores práticas de supervisão bancária, buscando mecanismos para o fortalecimento dos sistemas financeiros, entre elas as regras de alocação de capital mínimo para as IF.

Em 1988, o BCBS publicou o documento International Convergence of Capital Measurement and Capital Standards, que passou a ser conhecido como Acordo da Basileia, ou Basileia I. Ao definir que os bancos deveriam manter um capital maior ou igual a $8 \% \mathrm{em}$ relação ao seu total de ativos, o Acordo representou um significativo avanço rumo aos objetivos de estabelecer um nível adequado de capital nos sistemas bancários e garantir maior equidade competitiva entre os bancos (BCBS, 1988).

141 ISSN 2175-8069, UFSC, Florianópolis, v. 10, n. 21, p. 137-158, set./dez. 2013 
As grandes perdas ocorridas em diversas empresas como Barings Bank e Enron, decorrentes de fragilidades nos controles ou deficiências no gerenciamento das instituições, resultou, em 2004, na publicação da versão final do documento "Convergência Internacional de Mensuração e Padrões de Capital: Uma Estrutura Revisada", também chamado de Basileia II, que consiste num robusto conjunto de princípios para o controle de riscos e construção de ferramentas de gerenciamento de riscos, principalmente crédito, mercado, liquidez e operacional que serão utilizadas por bancos e autoridades supervisoras no exame de políticas e práticas de gestão (BCBS, 2004).

O primeiro Acordo da Basileia já enfatizava os riscos de crédito e riscos de mercado (a partir de 1996). O novo acordo, além de introduzir o risco operacional, traz uma série de opções para alocação de capital para risco de crédito e operacional, permitindo as IF escolherem abordagens que são mais adequadas ao escopo de suas operações (BCBS, 2004).

Além das questões que envolvem alocação de capital por meio do Pilar I, Basileia II destaca também a importância das IF e supervisores darem a devida atenção ao segundo e terceiro pilares, pois é fundamental que os requisitos mínimos de capital sejam acompanhados de uma robusta implementação do Pilar II e observância do Pilar III, detalhados a seguir (BCBS, 2004):

Pilar I: Cálculo de Exigências de Capital Mínimo, que dispõe sobre os modelos de cálculo dos requerimentos de capital mínimo para os riscos de crédito, de mercado e operacional.

Pilar II: Supervisão Bancária prevê uma atuação preventiva e consistente da autoridade supervisora na mensuração dos riscos e na validação dos modelos, devendo tomar medidas apropriadas de supervisão e devem intervir antecipadamente para evitar que o capital de determinado banco fique abaixo dos níveis mínimos, devendo exigir uma medida reparatória rápida se o capital não for mantido ou restaurado.

Pilar III: Disciplina de Mercado, que dá ênfase à transparência e importância de divulgação de informações ao mercado, quanto à prestação de contas, para franquear ao público as informações relativas à exposição de riscos e às competências em gestão.

\subsection{O Pilar III - Disciplina de Mercado}

Neste estudo, os termos disclosure, divulgação e evidenciação serão utilizados como sinônimos.

Segundo o BCBS (2001, p. 1), o Pilar III reconhece que a disciplina de mercado tem potencial para reforçar os padrões de capital mínimo (Pilar I) e o processo de supervisão (Pilar II), e assim promover a segurança e a solidez dos bancos e sistemas financeiros. A disciplina de mercado impõe fortes incentivos aos bancos para conduzir seus negócios de maneira segura, sólida e eficiente, incluindo um incentivo para manter uma forte base de capital como um colchão contra potenciais perdas futuras decorrentes da exposição ao risco.

O BCBS (2000, p. 3) apoia fortemente as questões que envolvem maior transparência, maior divulgação e disciplina de mercado por entender que são ferramentas cada vez mais importantes de supervisão.

Bushman, Piotroski e Smith (2001) definem transparência como "a abrangente disponibilidade de informação relevante e confiável sobre o desempenho periódico, situação financeira, oportunidades de investimento, governança, valor e risco das empresas de capital aberto" (BUSHMAN, PIOTROSKI e SMITH, 2001, p. 1). 
Em consonância com as recomendações do Pilar III, o BACEN publicou em 24 de dezembro de 2009 a Circular n. 3.477 que dispõe sobre a obrigatoriedade de várias IF divulgarem informações referentes à gestão de riscos. As informações requeridas em um total de 46 são de caráter qualitativo (12) e quantitativo (34) e abrangem basicamente a estrutura de gerenciamento de riscos, patrimônio de referência, parcelas do patrimônio de referência exigido, o índice de Basileia, exposições a risco de crédito, instrumentos mitigadores, risco de crédito da contraparte, cessão de crédito, derivativos, entre outras.

Conforme disposto no Art. $15^{\circ}$ da Circular BACEN 3.477, essas informações devem estar disponíveis em um único local, de acesso público e de fácil localização, no sítio eletrônico da IF juntamente com as relativas à estrutura de gestão de risco. A IF deve ainda publicar, em conjunto com as demonstrações contábeis, a localização dessas informações no sítio eletrônico.

\subsection{Estudos Similares}

A divulgação de informações sobre gestão de riscos em Instituições Financeiras foi abordada em diversas pesquisas, que em quase sua totalidade foram estudos fundamentalmente descritivos, iniciando com as pesquisas publicadas pelo Comitê da Basileia (BCBS, 2001a, 2002 e 2003a).

As pesquisas do BCBS, que têm como objetivo levantar as práticas de divulgação dos bancos com sede em países membros do Comitê da Basileia para a promoção da disciplina de mercado e para o desenvolvimento do Pilar III do Basileia II, mostraram que o nível de divulgação melhorou nos anos de 1999, 2000 e 2001 atingindo, respectivamente, 57\%, 59\% e $63 \%$ dos itens.

No contexto dos bancos brasileiros, Xavier (2003), que buscou comparar o grau de transparência das demonstrações contábeis com vistas a também contribuir com o Basileia II, assinala que apenas $26 \%$ das informações pesquisadas são divulgadas pelos principais bancos brasileiros no ano de 2002.

No ano de 2007, Di Benedito e Silva realizaram um estudo de caso em três IF cujo objetivo foi analisar o nível de transparência destas no período de 2004 a 2005. Os resultados mostraram que houve uma evolução na divulgação das informações prestadas, mas que o percentual médio de divulgação dos itens pesquisados em relação às recomendações do Basileia II em seu Pilar III - Disciplina de Mercado foi de apenas 20\%.

Os estudos de Doornik et al. (2007), também em 2007, avaliaram o nível de aderência dos dez maiores bancos brasileiros aos requerimentos do Basileia II e observaram que, de 2003 a 2005, houve uma pequena evolução nos índices de divulgação, passando de $25 \%$ para $29 \%$.

Costa, Goldner e Galdi (2007) tiveram como objetivo identificar características dos maiores bancos brasileiros relacionadas a um maior nível de disclosure. Os autores concluíram que o índice de divulgação das instituições analisadas é baixo já que apenas os cinco primeiros colocados no ranking obtiveram um índice de divulgação acima de $50 \%$. Em relação às características, a independência do Conselho de Administração, o tamanho da empresa, possuir ações no mercado de capitais, participar em níveis de governança corporativa da BOVESPA e emitir ADRs na Bolsa de Nova Iorque são responsáveis por um maior nível de divulgação pelas IF analisadas.

143 ISSN 2175-8069, UFSC, Florianópolis, v. 10, n. 21, p. 137-158, set./dez. 2013 
Em um trabalho focado no risco de mercado, Goulart e Carvalho (2004) pesquisaram e analisaram o grau de evidenciação por quatro bancos brasileiros, além de um banco de origem estrangeira, pois estes possuíam práticas mais avançadas de evidenciação do risco de mercado. Seus estudos mostraram que entre 1997 e 2002, houve uma evolução na evidenciação de informações sobre risco de mercado, mas também concluiu que esta evidenciação é bastante incipiente, principalmente ao se comparar com as práticas internacionais. Pereira (2006) relata que o acordo de Basileia II delinea-se como um instrumento relevante no esforço para aumentar a segurança e a solidez do sistema financeiro nacional.

A divulgação do risco operacional foi objeto de pesquisa por Barroso, Lustosa e Moraes (2004), cujo objetivo foi apresentar o grau de evidenciação do risco operacional em bancos no Brasil à luz das recomendações do Comitê de Basileia no período de 2000 a 2003. Os resultados demonstraram que as IF pesquisadas divulgam, em média, $25 \%$ do total de itens considerados como ideal pelo Comitê da Basileia .

Alves (2005) também realizou estudos visando apresentar o nível de divulgação do risco operacional por bancos no Brasil no período de 2003 a 2004. O autor aponta que os níveis de divulgação dos bancos com controle nacional aumentaram de 17,13\% para 24,31\%. Entretanto, os índices são muito inferiores aos praticados pelos bancos do exterior, que alcançaram 77,78\% e 81,48\% no mesmo período de 2003 a 2004.

O trabalho de Andrade, Niyama e Santana (2009), além de analisar o nível de disclosure do risco operacional também analisou a divulgação do risco de mercado. $\mathrm{O}$ objetivo foi analisar os 20 maiores bancos no Brasil de 2003 a 2008, verificando a aderência às recomendações de Basileia. Os autores indicam que a maioria das IF pesquisadas não possui nível de aderência adequado às recomendações de divulgação pelo Comitê de Basileia, apesar de uma evolução no período de 2003 a 2008, justificada, segundo os autores, pelo fato de que muitas das instituições não possuíam nenhuma evidenciação desses riscos.

Dantas et al. (2010) avaliaram o grau de transparência do risco de crédito praticado pelas IF brasileiras à luz do Basileia II e ainda as variáveis que explicaram o nível de divulgação desse risco. Segundo os autores, o nível de divulgação do risco de crédito atende a menos de $40 \%$ dos itens requeridos, mas que o nível de divulgação apresentou um crescimento lento e constante no período pesquisado (2001 a 2008). Em relação às variáveis explicativas, foram confirmadas as hipóteses de que a vigência do Acordo de Basileia II modificou o padrão de evidenciação e de que os bancos com maior volume de ativos, com maior rentabilidade, menor imobilização e com ações na BOVESPA apresentam maior grau de transparência.

Por fim, em uma pesquisa relacionando disclosure e Governança Corporativa, Lanzana, Silveira e Fama (2004) investigaram a relação entre o nível de disclosure adotado por empresas brasileiras de capital aberto e suas variáveis relacionadas à estrutura de propriedade e do conselho de administração. Os resultados apontaram que um maior nível de disclosure por essas empresas se deve às variáveis de direitos de controle, direitos sobre o fluxo de caixa do acionista controlador, maior percentual de membros externos no conselho de administração, identidade do controlador, porte da empresa, alavancagem, desempenho e valor de mercado. 


\section{Hipóteses de Pesquisa}

A partir do referencial teórico e as relações sugeridas em estudos anteriores, este estudo busca verificar se as IF que estão mais expostas a riscos estão promovendo ações adequadas de transparência ao mercado, como estar aderente à Circular BACEN 3.477.

As seguintes hipóteses foram desenvolvidas:

$H_{1}=A s$ IF com maior exposição a riscos apresentam maior aderência à Circular BACEN 3.477.

Essa hipótese será testada por meio de variáveis independentes relativas ao rating da IF (RAT), índice de Basileia (IB) e exposição ao instrumento financeiro derivativo (DER).

Como o estudo também verificará o nível de aderência de IF de controle público e privado, uma segunda hipótese foi criada:

H2 = As IF com tipo de controlador privado apresentam maior aderência à Circular BACEN 3.477.

\section{Metodologia}

Para a análise das hipóteses e apuração dos resultados visando avaliar como as variáveis independentes estão relacionadas com o nível de divulgação das informações, utilizou-se de modelo de regressão linear múltipla por meio da seguinte equação:

$\mathrm{NDGRi}=\alpha+\beta 1 \ln \mathrm{TAMi}+\beta 2 \mathrm{TCO}+\beta 3 \mathrm{AUDi}+\beta 4 \mathrm{BOV} \mathrm{i}+\beta 5 \mathrm{ESTi}+\beta 6 \mathrm{RPLi}+\beta 7 \mathrm{ALVi}+\beta 8 \mathrm{RATi}+\beta 9 \mathrm{DERi}+\beta 10 \mathrm{IBi}+\varepsilon \mathrm{i}$

Em que:

- NDGR: Nível de divulgação de informações de gestão de riscos pelas IF;

- TAM: Logaritmo natural do valor do Ativo Total da IF ( LN Ativo Total);

- TCO: Variável dummy que identifica o tipo de controlador da IF (público ou privado);

- AUD: Variável dummy que identifica se a IF possui ou não o órgão estatutário Comitê de Auditoria em sua estrutura organizacional;

- BOV: Variável dummy que identifica se a IF possui ações listadas na BOVESPA;

- EST: Variável dummy que identifica se a IF é de controle estrangeiro ou que possui participação estrangeira;

- RPL: Rentabilidade da IF resultante da razão do lucro líquido do período pelo patrimônio líquido do ano anterior ao período $(R P L=L L 2010 / P L 2009)$;

- ALV: Endividamento da IF frente ao capital de terceiros ( ALV = AtivoTotal $P L)$;

- RAT: Classificação atribuída por Agências de Rating que expressa a capacidade da IF em honrar seus compromissos;

- DER: Exposição que a IF possui em instrumento financeiro Derivativo sendo expressa pela razão entre a soma dos valores do derivativo do Ativo e Passivo e o Ativo Total;

- IB: Indicador percentual que expressa o nível de exposição a riscos de uma IF em relação ao seu Patrimônio de Referência (PR).

\subsection{Amostra e Coleta de Dados}

145 ISSN 2175-8069, UFSC, Florianópolis, v. 10, n. 21, p. 137-158, set./dez. 2013 
A amostra foi composta de IF constantes do relatório BACEN "50 Maiores Bancos e o Consolidado do Sistema Financeiro Nacional" e participantes do "Consolidado Bancário I" que são conglomerados compostos de pelo menos uma instituição do tipo Banco Comercial ou Banco Múltiplo com carteira comercial e instituição do tipo Banco Comercial, Banco Múltiplo com carteira comercial ou Caixa Econômica.

As informações foram coletadas por meio da técnica de pesquisa documental em documentos de divulgação relativos à gestão de riscos, na data-base Dezembro/2010, nos sítios eletrônicos das IF, principalmente nas seções "Institucional", "Relação com Investidores", "Governança Corporativa" e "Gerenciamento de Riscos", considerando o que determina o Art. $15^{\circ}$ da Circular BACEN 3.477.

A amostra total do "Consolidado Bancário I" é de $101 \mathrm{IF}$, conforme detalhado na Figura 1, sendo 71 de controle nacional e 30 de controle ou com participação estrangeira.

Figura 1 - IF do Estudo, por ordem alfabética

\begin{tabular}{|c|c|c|}
\hline $\begin{array}{l}\text { BCO A.J. RENNER S.A. } \\
\text { BCO ABC BRASIL S.A. } \\
\text { BCO ALFA S.A. } \\
\text { BCO ARBI S.A. } \\
\text { BCO AZTECA DO BR S.A. } \\
\text { BCO BARCLAYS S.A. } \\
\text { BCO BBM S/A } \\
\text { BCO BM\&F } \\
\text { BCO BMG S.A. } \\
\text { BCO BNP PARIBAS BR S.A. } \\
\text { BCO BONSUCESSO S.A. } \\
\text { BCO BRADESCO S.A. } \\
\text { BCO BRASCAN S.A. } \\
\text { BCO BTG PACTUAL S.A. } \\
\text { BCO BVA S.A. } \\
\text { BCO CAIXA GERAL - BR S.A. } \\
\text { BCO CAPITAL S.A. } \\
\text { BCO CARGILL S.A. } \\
\text { BCO CEDULA S.A. } \\
\text { BCO CLASSICO S.A. } \\
\text { BCO CONFID. CÂMBIO S.A. } \\
\text { BCO COOP. DO BRASIL S.A. } \\
\text { BCO COOP. SICREDI S.A. } \\
\text { BCO CR2 S/A } \\
\text { BCO CREDIBEL S.A. } \\
\text { BCO CRÉDIT AGRIC. BR S.A. } \\
\text { BCO CREDIT SUISSE (BR) S.A. } \\
\text { BCO CRUZEIRO DO SUL S.A. } \\
\text { BCO DA AMAZONIA S.A. } \\
\text { BCO DA CHINA BR SA - BOC } \\
\text { BCO DAYCOVAL S.A. } \\
\text { BCO DE LA NAC. ARGENTINA } \\
\text { BCO DE LA PROV. B. AIRES } \\
\text { BCO DE LA REP. URUGUAY }\end{array}$ & $\begin{array}{l}\text { BCO TOKYO-MITSUB. UFJ S.A. } \\
\text { BCO DO BRASIL S.A. } \\
\text { BCO DO ESTADO DE SERGIPE S.A. } \\
\text { BCO DO ESTADO DO PARÁ S.A. } \\
\text { BCO DO EST. RIO GRANDE DO SUL } \\
\text { BCO DO NORDESTE BRASIL S.A. } \\
\text { BCO FATOR S.A. } \\
\text { BCO FIBRA S.A. } \\
\text { BCO FICSA S.A. } \\
\text { BCO GE CAPITAL S.A. } \\
\text { BCO GUANABARA S.A. } \\
\text { BCO INDUSTRIAL DO BRASIL S.A. } \\
\text { BCO INDUST. E COMERCIAL S.A. } \\
\text { BCO INDUSVAL S.A. } \\
\text { BCO INTERCAP S.A. } \\
\text { BCO INTERMEDIUM S/A } \\
\text { BCO JBS S.A. } \\
\text { BCO JOHN DEERE S.A. } \\
\text { BCO KDB DO BRASIL S.A. } \\
\text { BCO KEB DO BRASIL S.A. } \\
\text { BCO LUSO BRASILEIRO S.A. } \\
\text { BCO MATONE S.A. } \\
\text { BCO MÁXIMA S.A. } \\
\text { BCO MERCANTIL DO BRASIL S.A. } \\
\text { BCO MODAL S.A. } \\
\text { BCO MORADA S.A } \\
\text { BCO MORGAN STANLEY S.A. } \\
\text { BCO OPPORTUNITY S.A. } \\
\text { BCO PANAMERICANO S.A. } \\
\text { BCO PAULISTA S.A. } \\
\text { BCO PETRA S.A. } \\
\text { BCO PINE S.A. } \\
\text { BCO POTTENCIAL S.A. } \\
\text { BCO PROSPER S.A. }\end{array}$ & $\begin{array}{l}\text { BCO RABOBANK S.A. } \\
\text { BCO RANDON S.A. } \\
\text { BCO RENDIMENTO S.A. } \\
\text { BCO RIBEIRAO PRETO S.A. } \\
\text { BCO RURAL S.A. } \\
\text { BCO SAFRA S.A. } \\
\text { BCO SANTANDER S.A. } \\
\text { BCO SCHAHIN S.A. } \\
\text { BCO SEMEAR S.A. } \\
\text { BCO SOC. GENERALE S.A. } \\
\text { BCO SOFISA S.A. } \\
\text { BCO SUMITOMO MIT. S.A. } \\
\text { BCO TOPÁZIO S.A. } \\
\text { BCO TRIANGULO S.A. } \\
\text { BCO VOTORANTIM S.A. } \\
\text { BCO VR S.A. } \\
\text { BCO WESTLB DO BR. S.A. } \\
\text { BANESTES S.A. } \\
\text { BANIF } \\
\text { BCO. BRACCE S.A. } \\
\text { BPN BRASIL S.A. } \\
\text { BRB - BCO BRASILIA S.A. } \\
\text { CAIXA ECON. FEDERAL } \\
\text { CITIBANK N.A. } \\
\text { DEUTSCHE BANK S.A. } \\
\text { DRESDNER BANK BR S.A. } \\
\text { HSBC BANK BRASIL S.A. } \\
\text { ING BANK N.V. } \\
\text { ITAÚ UNIBANCO S.A. } \\
\text { JPMORGAN CHASE BANK } \\
\text { NATIXIS BR S.A. BCO MÚLT. } \\
\text { NBC BANK S.A. - BCO MÚLT } \\
\text { PARANÁ BCO S.A. }\end{array}$ \\
\hline
\end{tabular}

Fonte: Elaborada pelos autores. 


\subsection{Variáveis Dependente e Independentes}

Este estudo traz como variável dependente o "Nível de Divulgação de Informações de Gestão de Riscos" pelas IF, denominada NDGR, que foi construída especialmente a partir dos estudos de Lanzana, Silveira e Fama (2006), Costa, Goldner e Galdi (2007) e Dantas et al. (2010), cuja apuração se dará por meio de classificação (0 - não divulgou e 1 - divulgou) para cada requisito exigido pela Circular BACEN 3.477, em um total de 46, não nos cabendo analisar a qualidade da informação divulgada, mas apenas se a IF divulgou ou não os requisitos.

Assim, toda IF terá um NDGR próprio, sendo calculado pela equação NDGRi $=\sum$ Nota .

Importante ressaltar que para aqueles casos em que a IF informar a não existência ou o não tratamento do requisito exigido, este será considerado como divulgado. Tal critério fundamenta-se no objetivo principal da Circular BACEN 3.477 que é o de transparência ao mercado.

As variáveis independentes testadas neste estudo e detalhadas no item 4 têm como referência os estudos anteriores sobre divulgação de informações pelas IF, seguindo principalmente as linhas de pesquisas realizadas por Lanzana, Silveira e Fama (2006), Costa, Goldner e Galdi (2007) e Dantas et al. (2010), apuradas por meio do relatório BACEN "50 Maiores Bancos e o Consolidado do Sistema Financeiro Nacional", na data-base Dezembro/2010 .

As hipóteses de pesquisa se relacionam com as seguintes variáveis independentes:

Figura 2 - Associação entre as hipóteses e as variáveis independentes

\begin{tabular}{|c|c|c|c|c|}
\hline HIPÓTESES & \multicolumn{3}{|c|}{ H1 $_{1}$} & H2 $_{2}$ \\
\hline VARIÁVEIS & RAT & DER & IB & TCO \\
\hline
\end{tabular}

Fonte: Elaborada pelos autores.

\section{Análise dos Resultados}

Os resultados descritos a seguir foram apurados utilizando-se do modelo apresentado no item 4, sendo que a variável dependente NDGR foi apurada por meio de classificação ( 0 não divulgou e 1 - divulgou) para cada requisito exigido pela Circular BACEN 3.477, em um total de 46, não nos cabendo analisar a qualidade da informação divulgada, mas apenas se a IF divulgou ou não os requisitos exigidos.

As variáveis independentes foram apuradas por meio do relatório BACEN "50 Maiores Bancos e o Consolidado do Sistema Financeiro Nacional", na data-base Dezembro/2010.

\subsection{Apuração da Variável Dependente NDGR}

A variável dependente NDGR, que representa o nível de divulgação de informações relativas à gestão de riscos pelas IF, foi apurada em função dos requisitos exigidos pela Circular BACEN 3.477 e de acordo com o que determina o Art. $15^{\circ}$ da referida circular. 
A distribuição das frequências do NDGR das IF é demonstrada no histograma na Figura 3. Das 101 IF pertencentes ao Consolidado Bancário I, apenas 50 (49,5\%) divulgaram as informações no formato estabelecido no Art. $15^{\circ}$, quantitativo este que entendemos não representar uma limitação ao estudo pelo fato dessas IF responderem por $85,6 \%$ do ativo total da amostra, evidenciando a relevância das mesmas para o Consolidado Bancário I.

\section{Figura 3 - Histograma NDGR}

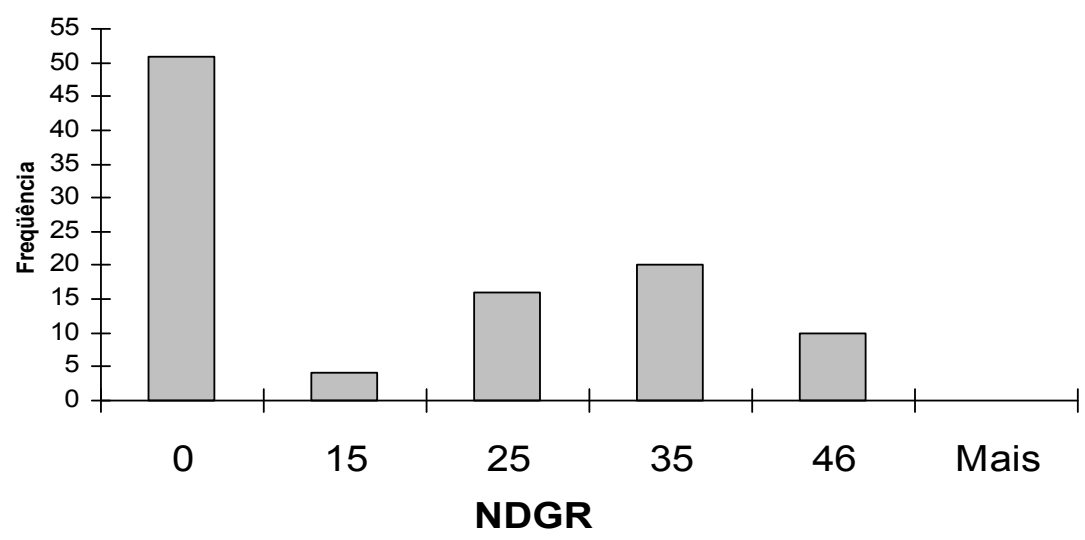

Fonte: Dados da pesquisa.

A composição do NDGR de toda IF pode ter sido afetada pela sua decisão em divulgar a não existência ou o não tratamento de requisitos exigidos pela Circular BACEN 3.477. Assim, para as IF que não citaram sobre os requisitos não tratados por ela tiveram os mesmos pontuados com " 0 - não divulgou", enquanto que para as IF que publicaram que não possuem ou não tratam determinados requisitos, tiveram os mesmos pontuados com "1 - divulgou". Entendemos que esse possível viés será gradativamente anulado nas próximas publicações, pois as IF que não tornaram público a não existência ou não tratamento dos requisitos, provavelmente o farão objetivando alcançar uma maior transparência.

Outra situação que pode ter afetado a composição do NDGR se refere à verificação por esta pesquisadora da divulgação ou não dos requisitos de caráter "qualitativo", que não se encontravam devidamente identificados nas publicações das IF. Entendemos, assim, que existe o viés da subjetividade da pesquisadora ao verificar se as informações qualitativas divulgadas atendiam ou não aos requisitos exigidos pela Circular BACEN 3.477.

\subsection{Divulgação por Tipo de Controlador}

Considerando apenas a amostra de 50 IF que divulgaram as informações relativas à Circular BACEN 3.477, sendo 7 de controle público e 43 de controle privado, a Figura 4 demonstra que as de controle público divulgaram mais informações que as de controlador privado. Já a Figura 5 nos mostra que, ao analisarmos por característica do requisito, os requisitos de caráter quantitativo são os mais divulgados, tanto nas IF de controle público quanto nas de controle privado. 
Figura 4 - Por Tipo de Controlador

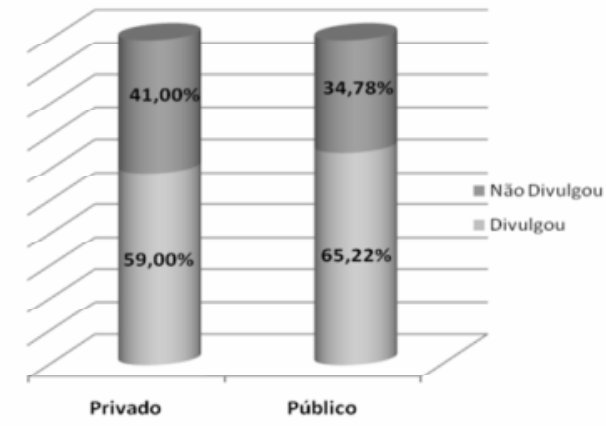

Fonte: Dados da pesquisa.
Figura 5 - Por Característica do Requisito

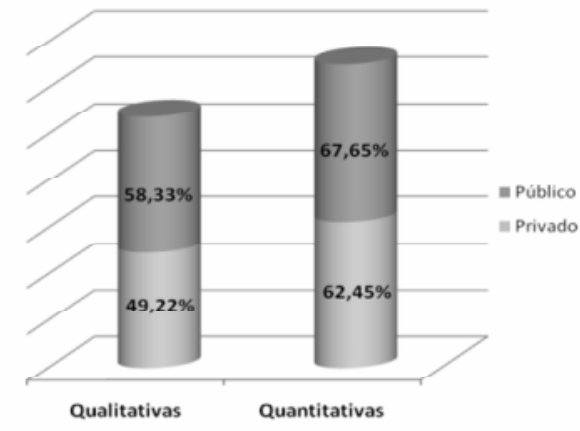

Fonte: Dados da pesquisa.

Interessante registrar também que, analisando os resultados dos requisitos divulgados, observamos que, tanto nos requisitos de caráter qualitativo quanto no quantitativo, a ordem dos mais divulgados assim como dos menos divulgados coincide, independente do tipo de controlador da IF.

Nos requisitos de caráter qualitativo, os mais divulgados são os requisitos relacionados à descrição dos objetivos e política de gerenciamento de riscos; metodologia para estabelecer limites às exposições sujeitas ao risco de contraparte e políticas de mitigação de risco; e estratégias e processos utilizados para o monitoramento contínuo da efetividade dos instrumentos de mitigação. O requisito referente à descrição dos objetivos e política de gerenciamento de riscos foi o único item qualitativo divulgado por todas as IF, fato que se justifica por ser ele uma condição sine qua non para a IF gerenciar riscos. Observa-se ainda que dentre os requisitos menos divulgados se encontram aqueles relativos à transferência de recursos entre o consolidado, liquidação antecipada de empréstimo e políticas de hedge.

Já nos requisitos de caráter quantitativo, observamos que nenhum deles foi divulgado por todas as IF e que os requisitos mais divulgados são os que tratam do valor total do PR; índice de Basileia (IB); valor da parcela do POPR, do PRE e o valor total do PRE. Dentre os menos divulgados, estão os relativos a hedge por meio de derivativos e derivativos para fins de intermediação, talvez pelo fato de muitas IF não se utilizarem de derivativos e quando utilizam, a exposição em relação ao ativo total não é significativa, em sua maioria.

\subsection{Estatística Descritiva das Variáveis}

As estimativas foram realizadas separadamente para a amostra de 50 IF que divulgaram os requisitos exigidos pela Circular BACEN 3.477 e para uma segunda amostra com 51 instituições que não divulgaram.

Analisando a variável dependente NDGR, observamos que das IF que divulgaram, obteve-se uma média de 27,54 pontos, sendo que a pontuação mínima obtida foi de cinco pontos e a máxima de 45 pontos. Podemos concluir então que nenhuma IF divulgou todos os requisitos da Circular BACEN 3.477, considerando o total de 46 requisitos (12 qualitativos e 34 quantitativos).

Em relação às variáveis independentes, podemos observar que a média da amostra das IF que divulgaram as informações é maior em quase a totalidade das variáveis, com exceção para as variáveis TCO $(0,86)$, fato evidenciado no item 5.2, RAT $(2,57)$ e IB $(36,50)$. Nesta última, entendemos ser coerente o comportamento da variável, pois as IF que possuem IB 
mais elevado tendem a não se preocuparem com ações de divulgação pública, por não apresentarem nível preocupante de exposição a riscos.

Tabela 1 - Estatística Descritiva das Variáveis

\begin{tabular}{l|r|r|r|r|r|r|r|r}
\hline \multirow{2}{*}{ Variável } & \multicolumn{2}{|c|}{ Média } & \multicolumn{2}{c|}{ Desvio-Padrão } & \multicolumn{2}{c|}{ Mínimo } & \multicolumn{2}{c}{ Máximo } \\
\cline { 2 - 8 } & \multicolumn{1}{|c|}{ Div. } & N.Div. & \multicolumn{1}{c}{ Div. } & N.Div. & \multicolumn{1}{c}{ Div. } & N.Div. & \multicolumn{1}{c}{ Div. } & N.Div. \\
\hline NDGR & 27.54 & 0 & 9.13 & 0 & 5 & 0 & 45 & 0 \\
TAM & $\mathbf{1 5 . 4 5}$ & 13.74 & 2.42 & 1.88 & 10.3 & 10.2 & 20.47 & 19.81 \\
TCO & 0.86 & 0.96 & 0.35 & 0.19 & 0 & 0 & 1 & 1 \\
EST & $\mathbf{0 . 3 4}$ & 0.27 & 0.47 & 0.45 & 0 & 0 & 1 & 1 \\
AUD & $\mathbf{0 . 4 8}$ & 0.21 & 0.50 & 0.41 & 0 & 0 & 1 & 1 \\
BOV & $\mathbf{0 . 3 2}$ & 0.11 & 0.47 & 0.32 & 0 & 0 & 1 & 1 \\
RPL & $\mathbf{0 . 1 4}$ & -0.06 & 0.58 & 0.70 & -0.40 & -4.82 & 4.16 & 0.56 \\
ALV & $\mathbf{8 . 3 3}$ & 8.23 & 5.06 & 11.89 & 1.08 & -9.64 & 31.28 & 63.21 \\
RAT & 2.57 & 3 & 1.19 & 1.16 & 1 & 1 & 5 & 5 \\
DER & $\mathbf{0 . 0 3}$ & 0.01 & 0.07 & 0.03 & 0 & 0 & 0.46 & 0.20 \\
IB & 36.50 & 45.28 & 46.14 & 93.72 & 10.89 & 4.3 & 204.89 & 618.12 \\
\hline
\end{tabular}

Fonte: Dados da pesquisa.

A Tabela 2 demonstra a correlação entre a variável dependente NDGR e as variáveis independentes da amostra total de 101 IF. A variável dependente NDGR e a variável independente "tamanho" (TAM) apresentaram uma correlação significativa $(0,50)$, constatando resultados de estudos anteriores em que se evidencia que IF maiores divulgam mais informações. Os resultados foram semelhantes ao apurarmos a correlação das variáveis para uma segunda amostra composta apenas das 50 IF que divulgaram os requisitos exigidos pela Circular BACEN 3.477.

Tabela 2 - Matriz de Correlação das Variáveis - Amostra Total - 101 IF

\begin{tabular}{c|r|r|r|r|r|r|r|r|r|r}
\hline & NDGR & \multicolumn{1}{|c|}{ TAM } & \multicolumn{1}{c|}{ TCO } & \multicolumn{1}{|c|}{ EST } & AUD & BOV & RPL & ALV & DER & IB \\
\hline NDGR & 1.00 & & & & & & & & & \\
TAM & 0.50 & 1.00 & & & & & & & & \\
TCO & -0.19 & -0.29 & 1.00 & & & & & & & \\
EST & 0.05 & 0.08 & 0.21 & 1.00 & & & & & & \\
AUD & 0.39 & 0.73 & -0.28 & -0.03 & 1.00 & & & & & \\
BOV & 0.29 & 0.49 & -0.42 & -0.25 & 0.42 & 1.00 & & & & \\
RPL & 0.24 & 0.47 & -0.37 & -0.08 & 0.36 & 0.28 & 1.00 & & & \\
ALV & 0.33 & 0.71 & -0.28 & -0.13 & 0.39 & 0.29 & 0.32 & 1.00 & & \\
DER & 0.29 & 0.51 & 0,26 & 0.24 & 0.21 & 0.09 & 0.13 & 0.37 & 1.00 & \\
IB & -0.11 & -0.43 & 0.09 & 0.27 & -0.20 & -0.11 & -0.17 & -0.72 & -0.30 & 1.00 \\
\hline
\end{tabular}

Fonte: Dados da pesquisa.

\subsection{Resultados das Regressões}

Considerando que nem todas as IF divulgaram informações relativas à variável rating (RAT), a Tabela 3 apresenta os resultados da aplicação do modelo de regressão para duas amostras, sendo a primeira para a amostra total de 101 IF e a segunda para a amostra de 66 IF 
que divulgaram a variável rating (RAT). Os resultados demonstram que independente da amostra, as variáveis independentes que apresentaram relação estatística significativa foram as mesmas, sendo elas: tamanho (TAM), alavancagem (ALV) e exposição a derivativos (DER).

Tabela 3 - Resultado das Regressões de Duas Amostras -Variável NDGR

\begin{tabular}{|c|c|c|c|c|}
\hline \multirow[t]{2}{*}{ VARIÁVEIS } & \multicolumn{2}{|c|}{$\begin{array}{c}\text { OLS - } 101 \text { IF - } \\
\text { AMOSTRA TOTAL }\end{array}$} & \multicolumn{2}{|c|}{ OLS - 66 IF - AMOSTRA COM "RAT " } \\
\hline & COEF. & P-VALOR & COEF. & P-VALOR \\
\hline Const & -39.66 & 0.004 & $-67,95$ & 0.022 \\
\hline TAM & 3.92 & 0.000 & 5.33 & 0.003 \\
\hline TCO & -2.42 & 0.697 & -2.92 & 0.632 \\
\hline EST & -2.27 & 0.506 & -1.80 & 0.732 \\
\hline AUD & 0.96 & 0.822 & 3.19 & 0.506 \\
\hline $\mathrm{BOV}$ & 0.48 & 0.925 & -0.09 & 0.988 \\
\hline RPL & 1.00 & 0.472 & -0.14 & 0.920 \\
\hline $\mathbf{A L V}$ & -0.39 & 0.000 & -0.42 & 0.001 \\
\hline RAT & - & - & 1.65 & 0.474 \\
\hline DER & 33.29 & 0.036 & 47.07 & 0.006 \\
\hline IB & 0.02 & 0.269 & 0.03 & 0.381 \\
\hline $\mathrm{R} 2$ & \multicolumn{2}{|c|}{0.3358} & \multicolumn{2}{|c|}{0.4138} \\
\hline
\end{tabular}

Fonte: Dados da pesquisa.

Buscando apurar melhor os resultados quanto ao possível viés da subjetividade desta pesquisadora na apuração do NDGR dos requisitos qualitativos, aplicamos o mesmo modelo de regressão para duas subdivisões da variável NDGR. Assim, analisamos também o nível de divulgação de informações qualitativas de gestão de riscos (NDGRQUALI) e o nível de divulgação de informações quantitativas de gestão de riscos (NDGRQUANTI).

Tabela 4 - Resultado das Regressões de Duas Amostras -Variável NDGRQUALI

\begin{tabular}{c|r|r|r|r}
\hline \multirow{2}{*}{ VARIÁVEIS } & \multicolumn{2}{|c|}{ OLS - 101 IF - } & OLS - 66 IF - AMOSTRA COM "RAT " \\
& \multicolumn{1}{|c|}{ AMOSTRA TOTAL } & \multicolumn{2}{|c}{ P-VALOR } \\
\cline { 2 - 5 } Const & COEF. & \multicolumn{1}{|c}{ P-VALOR } & COEF. & 0.005 \\
TAM & -10.83 & 0.003 & -19.74 & $\mathbf{0 . 0 0 1}$ \\
TCO & $\mathbf{0 . 9 9}$ & $\mathbf{0 . 0 0 0}$ & $\mathbf{1 . 4 4}$ & 0.842 \\
EST & -0.25 & 0.873 & -0.32 & 0.452 \\
AUD & -0.78 & 0.341 & -0.92 & 0.268 \\
BOV & -0.86 & 0.407 & 1.34 & 0.786 \\
RPL & -0.23 & 0.981 & -0.41 & 0.336 \\
ALV & $\mathbf{- 0 . 0 9}$ & 0.717 & -0.61 & $\mathbf{0 . 0 0 1}$ \\
RAT & - & $\mathbf{0 . 0 0 0}$ & $-\mathbf{0 . 1 1}$ & 0.310 \\
DER & 2.75 & -0.485 & 0.59 & 0.167 \\
IB & 0.00 & 0.190 & 5.60 & 0.136 \\
\hline R2 & \multicolumn{2}{|c|}{0.3526} & 0.01 & 0.4032 \\
\hline
\end{tabular}

Fonte: Dados da pesquisa. 
Tabela 5 - Resultado das Regressões de Duas Amostras -Variável NDGRQUANTI

\begin{tabular}{|c|c|c|c|c|}
\hline \multirow[t]{2}{*}{ VARIÁVEIS } & \multicolumn{2}{|c|}{$\begin{array}{c}\text { OLS - } 101 \text { IF - } \\
\text { AMOSTRA TOTAL }\end{array}$} & \multicolumn{2}{|c|}{ OLS - 66 IF - AMOSTRA COM "RAT " } \\
\hline & COEF. & P-VALOR & COEF. & P-VALOR \\
\hline Const & -28.83 & 0.008 & $-48,21$ & 0.041 \\
\hline TAM & 2.93 & 0.000 & 3.88 & 0.005 \\
\hline TCO & -2.16 & 0.649 & -2.60 & 0.577 \\
\hline EST & -1.48 & 0.581 & -0.88 & 0.835 \\
\hline AUD & 0.09 & 0.977 & 1.85 & 0.627 \\
\hline $\mathrm{BOV}$ & 0.51 & 0.896 & 0.32 & 0.943 \\
\hline RPL & 1.23 & 0.126 & 0.46 & 0.612 \\
\hline ALV & -0.29 & 0.000 & -0.31 & 0.002 \\
\hline RAT & & & 1.05 & 0.563 \\
\hline DER & 30.53 & 0.023 & 41.46 & 0.004 \\
\hline IB & 0.01 & 0.303 & 0.01 & 0.504 \\
\hline $\mathrm{R} 2$ & & & & \\
\hline
\end{tabular}

Fonte: Dados da pesquisa.

Os resultados de todas as regressões demonstram que as variáveis que apresentaram relação estatística significativa não se diferem e que a não presença de relação estatística significativa da variável Derivativos (DER) na Tabela 4 é bastante coerente pelo fato de que o NDGRQUANTI é fortemente influenciado por requisitos específicos de exposição ao instrumento financeiro Derivativos exigidos pela Circular BACEN 3.477, o que não acontece com o NDGRQUALI.

Considerando o relacionamento entre as hipóteses de pesquisa e as variáveis independentes, passamos a detalhar os resultados com foco em identificar as variáveis determinantes para um maior nível de divulgação de informações de gestão de riscos pelas IF.

A hipótese $\mathrm{H}_{1}$ assume a premissa de que as IF com maior exposição a riscos divulgam mais informações, ou seja, apresentam maior aderência à Circular BACEN 3.477, sendo testada pelas variáveis rating (RAT), derivativos (DER) e índice de Basileia (IB).

Observamos que a variável derivativos (DER) apresentou relação estatística significativa e demonstra uma relação positiva com a variável dependente NDGR, indicando que quanto maior o nível de exposição ao instrumento derivativo, maior o nível de divulgação de informações. Esse resultado corrobora a hipótese $\mathrm{H}_{1}$, além de estar alinhado com as recomendações do BCBS em que IF com exposições significativas a riscos devem aumentar o seu nível de divulgação pública, oferecendo aos participantes de mercado informações acerca de sua estrutura e instrumentos mitigadores dos riscos, demonstrando sua capacidade de absorver perdas financeiras (BCBS, 2001). As demais variáveis de teste da hipótese $\mathrm{H}_{1}$ não apresentaram relação estatística significativa. Em relação à variável $\mathrm{IB}$, esse resultado foi também evidenciado pelos estudos de Dantas et al. (2010), em que a variável IB não se mostrou relevante para explicar um maior nível de divulgação por parte de IF.

Em relação à hipótese $\mathrm{H}_{2}$, observamos que a variável relacionada ao tipo de controlador da IF (TCO) não apresentou relação estatística significativa, ou seja, não há evidências de que IF com controlador privado divulguem mais informações do que as de controle público. Assim, a hipótese $\mathrm{H}_{2}$ foi rejeitada. Situação semelhante foi verificada nos 
estudos de Dantas et al. (2010). Já nos estudos de Lanzana, Silveira e Fama (2006), essa variável se mostrou significante, mas temos de considerar que a amostra era de companhias abertas não financeiras.

Quanto às demais variáveis independentes (TAM, AUD, BOV, EST, RPL e ALV) que não foram relacionadas às hipóteses do estudo, verificamos pelos resultados da regressão que duas delas (TAM e ALV) apresentaram relação estatística significativa para explicar o nível de divulgação das IF.

A variável tamanho (TAM), que representa o tamanho da IF de acordo com seu Ativo Total, apresentou evidências de relação estatística significativa e com relação positiva, indicando que quanto maior a IF, maior o nível de divulgação das informações. Esses resultados estão alinhados com os estudos anteriores, tais como os de Lanzana, Silveira e Famá (2006), Costa, Goldner e Galdi (2007) e Dantas et al. (2010).

Já a variável alavancagem (ALV), que caracteriza o endividamento da IF frente ao capital de terceiros, apresentou relação estatística significativa com uma relação negativa com a variável dependente NDGR, indicando que quanto mais alavancada a IF menor o nível de divulgação das informações.

Em resumo, o estudo apresentou evidências de que IF com maior exposição a riscos apresentam maior aderência à Circular BACEN 3.477, confirmando a hipótese de pesquisa $\mathrm{H}_{1}$ e corroborando os esforços do BCBS no sentido de promover uma maior divulgação pública principalmente para IF com exposições significativas a riscos. Adicionalmente, não houve evidências de que IF com controlador Privado divulguem mais informações do que as de controle Público, rejeitando-se a hipótese $\mathrm{H}_{2}$ e ainda que as variáveis independentes tamanho (TAM) e alavancagem (ALV) apresentaram relação estatística significativa para explicar o nível de divulgação de informações de gestão de riscos requeridas pela Circular BACEN 3.477 .

\section{Considerações Finais}

Este estudo teve como objetivo verificar o nível de divulgação das IF e as variáveis determinantes para um maior nível de divulgação de informações inerentes à gestão de riscos, tendo como referência os requisitos exigidos pela Circular BACEN 3.477 de dezembro de 2009 que dispõe sobre a obrigatoriedade de IF divulgarem informações referentes à gestão de riscos, ao Patrimônio de Referência Exigido - PRE, tratado pela Resolução n. 3.490 de agosto de 2007 e à adequação do Patrimônio de Referência - PR, de que trata a Resolução n. 3.444 de fevereiro de 2007.

O pressuposto foi de que as IF que possuem maior exposição a riscos apresentariam maior aderência à Circular BACEN 3.477.

Da amostra de 101 IF participantes do "Consolidado Bancário I" do BACEN, apenas $50(49,5 \%)$ divulgaram as informações relativas à Circular BACEN 3.477 nos padrões definidos pelo seu Art. $15^{\circ}$, representando 85,6\% do Ativo Total da amostra, evidenciando a relevância das IF que divulgaram para o Consolidado Bancário I.

Em relação aos dois objetivos principais propostos para o estudo: (i) verificar o nível de aderência das IF às exigências de divulgação de informações requeridas pela Circular BACEN 3.477 e (ii) identificar as variáveis determinantes de um maior nível de divulgação dessas informações pelas IF, compreendemos que os mesmos foram atendidos.

153 ISSN 2175-8069, UFSC, Florianópolis, v. 10, n. 21, p. 137-158, set./dez. 2013 
Nas questões que envolvem o nível incipiente de divulgação de informações relativas à gestão de riscos por parte de IF, os resultados apurados estão condizentes com o referencial teórico apresentado. Entretanto, as evidências encontradas contradizem as expectativas de que o nível de divulgação por parte das IF no Brasil teria uma evolução bastante significativa pelo fato deste estudo tratar de requisitos de divulgação determinados por órgão supervisor e não de decisões voluntárias de divulgação. Nesse sentido, os resultados encontrados neste artigo estão em linha com os resultados de Dantas et al. (2010), que mostraram que o grau de evidenciação do risco de crédito nas divulgações qualitativas e quantitativas requeridas por Basileia II para os Bancos atuantes no mercado brasileiro sofreu aumento de forma constante e paulatina entre o primeiro trimestre de 2001 e o quarto de 2008, mas estava distante do padrão requerido por Basileia II.

No que tange às variáveis determinantes de um maior nível de divulgação, o estudo apresentou evidências de que as variáveis tamanho (TAM), derivativos (DER) e alavancagem (ALV) apresentaram relação estatística significativa com a variável dependente NDGR, com uma relação negativa para esta última variável.

Quanto às questões relativas ao nível de divulgação de acordo com o tipo de controlador, verificamos que as IF de controle público $(65.22 \%)$ divulgaram mais informações que as de controlador privado (59\%) e que, em ambos, as informações de caráter quantitativo são as mais divulgadas.

Quanto às duas hipóteses do estudo, em que $\mathrm{H}_{1}$ assume a premissa de que as IF com maior exposição a riscos divulgam mais informações, ou seja, apresentam maior aderência à Circular BACEN 3.477 e $\mathrm{H}_{2}$ de que IF com tipo de controlador privado apresentam maior aderência à Circular BACEN 3.477, o estudo corroborou apenas a hipótese $\mathrm{H}_{1}$, por meio da relação estatística significativa apresentada pela variável derivativos (DER), uma de suas variáveis de teste. Esse resultado está alinhado com as diretrizes do BCBS em que IF com exposições significativas a riscos devem aumentar o seu nível de divulgação pública, oferecendo aos participantes de mercado informações acerca de sua estrutura e instrumentos mitigadores dos riscos, demonstrando sua capacidade de absorver perdas financeiras. (BCBS, 2001).

Os resultados deste estudo se referem exclusivamente a 101 IF participantes do Consolidado Bancário I do BACEN e apresenta como potencial limitação o período de pesquisa. A data-base dezembro de 2010 caracteriza a primeira data-base exigida para divulgação das informações requeridas pela Circular BACEN 3.477. A variável dependente NDGR poderá ter sido afetada por ser a primeira data-base com obrigatoriedade de divulgação, apesar de verificado nos estudos anteriores que várias IF já praticam a divulgação de informações buscando estar em conformidade com as diretrizes do Pilar III - Basileia II.

Cabe ressaltar que os resultados apresentados apontam para possibilidades significativas de incremente e melhoria no disclosure de informações relacionadas à gestão de risco pelos bancos atuantes no mercado brasileiro.

Com base nos resultados deste estudo, sugerimos, para pesquisas futuras, que seja analisada comparativamente a evolução do nível de divulgação em relação à Circular BACEN 3.477 considerando as futuras datas-base de divulgação, conforme estabelecido no Art. $14^{\circ}$, com a aplicação de modelo de painéis e que sejam investigados os motivos da não divulgação das informações por 50,5\% das IF da amostra, apesar de se tratar de uma exigência do órgão supervisor.

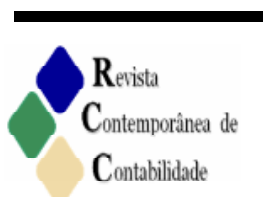




\section{Referências}

ALVES, C. A. M. A Divulgação do Risco Operacional segundo Recomendações do Comitê da Basileia: Estudo Em Bancos com Carteira Comercial no Brasil. 2005. Dissertação (Mestrado em Administração) - Centro de Pesquisa e Pós-Graduação em Administração, Departamento de Administração da Universidade Federal do Paraná, Curitiba, 2005.

ALVES, C. A. M.; ALVES, N. T. H. Análise do Nível de Divulgação da Governança Corporativa Segundo Recomendações do Comitê da Basileia: Um Estudo em Bancos Privados e Públicos Brasileiros. Revista Universo Contábil, v. 5, n. 1, p. 06-21, jan./mar. 2009.

ANDRADE, L. F. F. de; NIYAMA, J. K.; SANTANA, C. M. Nível de "Disclosure” Sobre Riscos de Mercado e Operacional: Uma análise comparativa dos vinte maiores bancos brasileiros. In: CONGRESSO USP DE CONTROLADORIA E CONTABILIDADE, 9, 2009, São Paulo. Anais... São Paulo: Congresso USP, 2009.

\section{BANCO CENTRAL DO BRASIL. 50 maiores bancos e o consolidado do sistema financeiro nacional.}

BANCO CENTRAL DO BRASIL. Resolução 3.380, de 29 de junho de 2006. Dispõe sobre a implementação de estrutura de gerenciamento do risco operacional. Diário Oficial [da] República Federativa do Brasil, Brasília, 29 jun. 2006.

BANCO CENTRAL DO BRASIL. Resolução 3.464, de 26 de junho de 2007. Dispõe sobre a implementação de estrutura de gerenciamento do risco de mercado. Diário Oficial [da] República Federativa do Brasil, Brasília, 26 jun. 2007.

BANCO CENTRAL DO BRASIL. Resolução 3.721, de 30 de abril de 2009. Dispõe sobre a implementação de estrutura de gerenciamento do risco de crédito. Diário Oficial [da] República Federativa do Brasil, Brasília, 30 abr. 2009.

BANCO CENTRAL DO BRASIL. Circular 3.477, de 30 de abril de 2009. Dispõe sobre a divulgação de informações referentes à gestão de riscos, ao Patrimônio de Referência Exigido (PRE), de que trata a Resolução n. 3.490, de 29 de agosto de 2007, e à adequação do Patrimônio de Referência (PR), de que trata a Resolução n. 3.444, de 28 de fevereiro de 2007.

BANCO CENTRAL DO BRASIL. Manual de Supervisão Bancária. Disponível em: $<$ https://www3.bcb.gov.br/gmn/visualizacao/listarDocumentosManualPublico.do?method=list arDocumentosManualPublico\&idManual=1>. Acesso em: 20 abr. 2013

BARROSO, M. O.; LUSTOSA, P. R. B.; MORAES, M. C. Disclosure de risco operacional em bancos no Brasil: evidências preliminares à luz do Novo Acordo da Basileia . In: CONGRESSO USP DE CONTROLADORIA E CONTABILIDADE, 4, 2004, São Paulo. Anais. São Paulo: Congresso USP, 2004. 
BARTH, J.; CAPRIO, G.; AND LEVINE, R. Bank Regulation and Supervision: What Works Best? Univ. of Minnesota, Carlson School of Management; World Bank Policy Research Working Paper n. 2725. 2001. Disponível em: $<$ http://ssrn.com/abstract $=269488$ or http://dx.doi.org/10.2139/ssrn.269488>. Acesso em: 01 mar.2013.

BASEL COMMITTEE ON BANKING SUPERVISION (BCBS). International Convergence of Capital Measurement and Capital Standards. BIS, 1988.

BASEL COMMITTEE ON BANKING SUPERVISION (BCBS). A New Capital Adequacy Framework: Pillar 3 Market Discipline. BIS, 2000.

BASEL COMMITTEE ON BANKING SUPERVISION (BCBS). Working Paper on Pillar 3 - Marketing Discipline. BIS, 2001.

\section{BASEL COMMITTEE ON BANKING SUPERVISION (BCBS). International}

Convergence of Capital Measurement and Capital Standards: A Revised Framework. Comprehensive Version. Basel: BIS, 2006.

BASEL COMMITTEE ON BANKING SUPERVISION (BCBS). Public Disclosures by Banks: Results of the 1999 Disclosure Survey. Basel: BIS, 2001a, 2002 e 2003a.

BERNSTEIN, P. Desafio aos Deuses - A Fascinante História do Risco, Ed. Campus, 1997.

BEYER, A.; COHEN, D. A.; LYZ, T. Z.; WALTHER, B. R.; The Financial Reporting environment: Review of the recent literature? Journal of Accounting Economics. V. 50, n. 2-3, p. 296-343, 2010.

BUSHMAN, R.; PIOTROSKI, J.; SMITH, A. What determines corporate transparency? Working Paper. University of Chicago, 2001.

CANNATA, F.; QUAGLIARIELLO, M. The Role of Basel II in the Subprime Financial Crisis: Guilty or Not Guilty? CAREFIN Research Paper n. 3/09. Available at SSRN: $<$ http://ssrn.com/abstract=1330417>. Acesso em: 14 jan. 2009.

CHEROBIM, A. P. M. S.; ALVES, C. A. M. Investigação Sobre Evidenciação do Risco Operacional: Estudo Multicaso em Instituições Financeiras Brasileiras. Revista de Administração e Contabilidade da Unisinos. V. 3, n. 2, p. 124-138, 2006.

COSTA, Fábio Moraes da; GOLDNER Fábio; GALDI, Fernando Caio. Análise dos Fatores que Influenciam no Disclosure dos Maiores Bancos Brasileiros. In: CONGRESSO USP DE CONTROLADORIA E CONTABILIDADE, 7, 2007, São Paulo. Anais... São Paulo: Congresso USP, 2007.

CRESWELL, J. W. Projeto de Pesquisa: método qualitativo, quantitativo e misto. Porto Alegre: Artmed, 2007. 
CRUZ, C. V. O.; LIMA, G. A. S. F. de. Reputação Corporativa e Nível de Disclosure das Empresas de Capital Aberto no Brasil. Revista Universo Contábil. V. 6, n. 1, p. 85-101, jan./mar. 2010.

DANTAS, José Alves; ZENDERSKY, Humberto Carlos; SANTOS, Sérgio Carlos dos;

NIYAMA, Jorge Katsumi. A Dualidade entre os Benefícios do Disclosure e a Relutância das Organizações em aumentar o Grau de Evidenciação. E \& G Economia e Gestão. Belo Horizonte, v. 5, n. 11, p. 56-76, dez 2005.

DANTAS, José Alves; RODRIGUES, Fernanda Fernandes; RODRIGUES, Jomar Miranda; CAPELLETTO, Lucio Rodrigues. Determinantes do grau de evidenciação de risco de crédito pelos bancos brasileiros. Revista Contabilidade e Finanças. V. 21, n. 52, São Paulo, 2010.

DI BENEDITO, E. A.; SILVA, R. N. Análise do nível de transparência das IF no Brasil em Relação ao Novo Acordo de Capitais (Basileia II). ENANPAD, 31, 2007, Rio de Janeiro. Anais... Rio de Janeiro: ANPAD, 2007.

DOORNIK, B. F. N. V.; SILVA, E. B. da; CAPELLETTO, L. R.; LHAMAS, R. C. G. Transparência das demonstrações contábeis após o acordo de Basileia 2: um estudo temporal dos 10 maiores bancos do país. ENANPAD, 31, 2007, Rio de Janeiro. Anais... Rio de Janeiro: ANPAD, 2007.

DUARTE JÚNIOR, A. M; VARGA, Gyorgy (Organizadores). Gestão de Riscos no Brasil. Rio de Janeiro: Financial Consultoria, 2003.

GOULART, André M. Cintra; CARVALHO, L. Nelson. Evidenciação Contábil do Risco de Mercado por IF no Brasil. UNB Contábil. V. 7, n. 1, Brasília, 2004.

GUJARATI, Damodar N. Econometria Básica. São Paulo, 2000.

LANZANA, Ana Paula; SILVEIRA, Alexandre Di Miceli da; FAMÁ, Rubens. Existe Relação entre Disclosure e Governança Corporativa no Brasil? ENANPAD, 30, 2006, Salvador. Anais... Salvador: ANPAD, 2006.

PEPPE, Márcio Serpejante. O novo acordo da Basileia. São Paulo: BDO Trevisan, 2006.

PEREIRA, José Matias. Gestão do Risco Operacional: Uma avaliação do novo acordo de capitais - Basileia II. Revista Contemporânea de Contabilidade. V. 1, n. 6, 2006.

LEUZ, C.; WYSOCKI, P. Economic Consequences of Financial Reporting and Disclosure Regulation: What have we Learned? Working Paper. SSRN. 2008.

WOOLDRIDGE, Jeffrey M. Introdução à Econometria: Uma abordagem Moderna. São Paulo, 2006. 
XAVIER, P. H. M. Transparência das Demonstrações Contábeis dos Bancos no Brasil:

Estudo de Caso sob a Perspectiva do Acordo "Basileia 2". Dissertação (Mestrado em Ciências Contábeis) - Faculdade de Economia, Administração e Contabilidade, Universidade de São Paulo, São Paulo, 2003. 